\section{EVOLUTION IN ASTRONOMY*}

\author{
By SiR JAMES JEANS, O.M., F.R.S.
}

NE of the most important, and also one of the most fascinating, of the problems of astronomy is the tracing out of the steps by which the universe bas evolved from primitive beginnings to its present complex form. But until recently it was like trying to piece together a jig-saw puzzle from which some of the more important pieces were missing. Happily the situation has changed rapidly of late; more pieces of the puzzle have come to light, and one in partioular-a better knowledge of the possible sources of stellar energy - is found to fit very convincingly into a big vaeant gap, with the result that the whole picture begins to assume a logical and satisfying shape.

Most cosmogonies have assumed that the universe began in a state of complete chaos. Newton saw that a mass of chaotic matter spread uniformly through space would form into condensations under its own gravitational forces, and that these would ultimately divide into separate detached masses. He here indicated the first broad lines of cosmic evolution; we are now able to fill in many of the details.

Astronomers have recently made two complementary discoveries which fit together very satisfactorily - that our galactic system of stars is smaller in size than used to be thought, and that the extra-galactic nebulæ are larger. Obscuring matter spread through space dims the brightness of the stars, making them appear more distant than they are; the recognition of this has brought the distant stars nearer and the galactic system has shrunk. Simultaneously, photometric studies of the sky have shown that the extra-galactic nebulæ extend much farther than appears on the photographs from which we used to estimate their dimensions. It is now clear that the galactic system and the extra-galactic nebulæ are all about the same size, their longest diameters perhaps averaging 100,000 light-years.

Their masses also are found to be not very dissimilar. The mass of the galactic system can be determined from the gravitational pull it exerts on the sun and the stars in its proximity; it proves to be rather more than 100,000 million times the mass of the sun. The masses of the extra-galactic nebulæ can be estimated from the gravitational pulls they exert on one another or on other moving objects; estimates of nebular masses range from 20,000 million to 200,000 million suns.

In this way the galactic system and the extragalactic nebulæ prove to be similar objects, which we may conveniently describe as 'galaxies'. They are scattered fairly uniformly through space at an average distance apart of perhaps a million lightyears. If we assume their average mass to be 100,000 million suns, the average density of matter in space is $2.4 \times 10^{-28}$, which gives 200 atoms of hydrogen, or one of mercury, to the cubic metre. If the universe is really expanding in the way suggested by the spectra of the nebulæ, this density is continually decreasing, its value being halved every 450 million years. But the density of the primeval chaos must have been at least of the order just mentioned. With this density adjacent molecules are about a foot apart, while the free-path must be meesured in light-years.

- Substance of a course of lectures delivered at the Royal Institution on November 3,10 and 17.
If we know the density of a distribution of chaotic matter, and the average speed of the thermal motion of its units, we can calculate the average mass of the condensations which will form in it. Newton conjectured that these condensations would be of stellar mass ; we now know they would be of galactic mass. Condensations having an average mass of 100,000 million suns would result from a density of $2 \cdot 4 \times$ $10^{-28}$ and a thermal velocity of $37 \mathrm{~km}$. a second. The thermal velocity might easily have this high value if the chaos contained a good proportion of free electrons, since these have a speed of $111 \mathrm{~km}$. a second at $0^{\circ} \mathrm{C}$.

Thus the first step in the evolution of the universe was probably the breaking up of a primeval chaos into the separate masses which now form the galaxies. These are all of the shapes which would be assumed by compressible masses in rotation, and rotation can be observed spectroscopically in the nearer nebuli. We do not know how it originated-possibly from currents and swirls in the primeval chaos. In any event, as the condensations contracted under their own gravitation, their speeds of rotation would increase and they would assume in turn the shapes appropriate to different rates of rotation. In the last stage of all, matter lies spread out in the equatorial plane of the rotating mass, where it again becomes liable to gravitational break up, and will form detached condensations. On inserting reasonable values for the density and thermal velocity of this matter, we find that the condensations will be of stellar mass. Thus, except for the intermediate step of the formation of galaxies, we can picture the stars as having been formed much as Newton imagined. As the average density of the parent nebula would scarcely be greater than $10^{-22}$, the new-born stars will be of very low density; they will perhaps exist as distinct and detached masses while their densities are still as low as $10^{-20}$.

They will of course begin by contracting rapidly under their own gravitation; a mathematical theorem of Poincaré helps us to follow their progress. It tells us that the total thermal energy imprisoned in a gaseous star at any time is just equal to the total energy which the star has lost, or would have lost, in contracting from a state of infinite diffusion. Incidentally, we notice that the more a gaseous star loses energy, the hotter it becomes-not the cooler, as we might be tempted to think.

Thus when the size and composition of a star is known, we can calculate its total thermal energy, and so deduce the temperature of its interior. It is found that the average temperature of the sun's interior must be about $20,000,000^{\circ}$, unless the sun contains a large amount of hydrogen, in which case it will be substantially lower. When other stars are treated in the same way, the surprising result emerges that for the largest class of known stars-the 'mainsequence' stars-the average internal temperature must be exactly or very nearly the same as for the sun.

The total energy supplied by gravitational contraction can, as is well known, only provide a small fraction of the total energy which the sun must have radiated even in geological times, and it has for some time been suspected that the sun-and of course the other main-sequence stars also-must draw energy from some source which comes into operation as soon as stellar matter attains a temperature of the order of $20,000,000^{\circ}$. The recent intensive study of nuclear physics has revealed just such a source of energy.

In a famous series of experiments, Rutherford 
showed that the nuclei of the atoms of the light elements can be transmuted into nuclei which are chemically different through bombardment by $\alpha$ particles. Now at the high temperatures of stellar interiors, $\alpha$-particles, which are merely helium nuclei, are a normal constituent of stellar matter, the helium atoms being broken up into their constituent particles by the heat. The stars become alchemical laboratories, in which the chemical elements continually suffer transmutation as their atoms or nuclei are bombarded by $\alpha$-particles and other swiftly moving projectiles-protons, neutrons, deuterons, and the like.

Reactions of this type are called thermonuclear. They are mostly very sensitive to changes of temperature, so that each reaction may be associated with a definite critical temperature; below this temperature the reaction occurs only in minute amounts, but above it in torrential amounts. This critical temperature depends very much on the complexity of the nuclei involved in the reaction, being approximately proportional to the square of the atomic numbers of these nuclei. It is consequently lowest-less than $1,000,000^{\circ}$-for the simple reaction of one proton with another. In this reaction a deuteron is formed, and as this leaves an excess of positive electricity, a positron is set free. The deuteron may afterwards capture two more protons and form a helium nucleus.

Next, at temperatures of from 3 to 7 million degrees, protons may react with the nuclei of the light elements lithium, beryllium and boron.

Then, at temperatures of the order of $20,000,000^{\circ}$, comes the reaction of a proton with a carbon nucleus of mass 12. The combination forms a nitrogen nucleus of mass 13, but this is only the first of a series of processes. The nitrogen nucleus may capture a second proton, becoming an ordinary nitrogen nucleus of mass 14, and then a third proton, becoming a nitrogen nucleus of mass 15. This may capture yet a forirth proton, but the result is not a nitrogen nucleus of mass 16 ; it is usually a carbon nucleus of mass 12 , together with a helium nucleus, or $\alpha$-particle, of mass 4. Actually this description has omitted subsidiary processes which do not affect the final result. The main events consist of the carbon nucleus swallowing four protons in succession, and thereby being pushed along the sequence of nitrogen isotopes until this road comes to an end. It then resumes the form of the carbon nucleus with which it started, the four protons it has swallowed being disgorged in the form of a helithm nucleus. In this way four protons are bound together to form a helium nucleus, all the other nuclei emerging unaltered-the carbon has merely acted as a catalyst.

This transmutation may not appear to have any relation to the supply of energy for radiation, until we notice that the total mass of the matter concerned has experienced a diminution. The atomic weight of hydrogen $(\mathrm{O}=16)$ is 1.008 , whence the mass of four protons is $4 \cdot 030$, while the mass of the resulting helium nucleus is only $4 \cdot 003$. Thus there has been a loss of mass of 0.027 units, or one part in 150 of the total mass involved.

We know that all radiation has mass associated with it at the fixed rate of $1 \mathrm{gm}$. for every $9 \times 10^{20}$ ergs of radiant energy. Thus the sun, radiating $3.8 \times 10^{33}$ ergs a second, must lose $4.2 \times 10^{12} \mathrm{gm}$. a second-250 million tons a minute. The core of the problem of the source of solar radiation is the finding of mass of this amount inside the sun which is capable of being set free in the form of radiation. Now each thermonuclear reaction in which mass is lost results in an equivalent emission of radiation in the form of $\gamma$ - or X-rays; these are soon absorbed by the stellar matter and re-emitted as temperature radiation in equilibrium with the stellar matter. Thus thermonuclear processes are fully capable of explaining the sun's radiation qualitatively.

They are also adequate quantitatively. To emit its present stream of radiation for a period of 2,000 million years, the sun must lose one part in 7,500 of its mass ; if it consisted wholly of hydrogen (apart from a small amount of carbon acting as a catalyst) it could transform one part in 150 of its mass into radiation by the process just considered. A sun in which only one fifth of one per cent was hydrogen could provide the present sun's radiation for 2,000 million years-and it is fairly certain that the sun contains more hydrogen than this. The evidence of astrophysics is that hydrogen is the most abundant element in most stars.

Other classes of stars present different problems, to which different answers must be given. 'The most luminous stars of all, apart from freaks, emit about 7,500 times as much radiation per gram of their mass as the sun. Neither thermonuclear reactions nor anything else could enable these stars to radiate at their present rates for 2,000 million years; to do this they would have to transform the whole of their mass into radiation. We must conclude that such stars cannot radiate as at present for another 2,000 million years, and probably have not done so in the past, unless they can draw on external sources of energy, as, for example, by an accretion of matter from outside. The red giants and Cepheid variables, which do not belong to the main sequence, have central temperatures well below the $20,000,000^{\circ}$ which characterizes the main sequence stars. The carbon reaction cannot occur in these in appreciable amounts, but it seems likely that reactions with elements lighter than carbon may provide the energy for their radiation. In time, all these light elements will have been used up; the star will then contract, its internal temperature continually increasing, until it reaches $20,000,000^{\circ}$. The star has now joined the main sequence, and the carbon reaction comes into play to supply it with radiation.

Again, the smallest stars have central temperatures well above the critical $20,000,000^{\circ}$. These must have used up all their hydrogen, and then started contracting afresh. The energy they are emitting as radiation may be derived from their gravitational contraction -which could supply radiation for perhaps 100,000 million years-or possibly from some other thermonuclear processes, not yet identified, which occur at temperatures above $20,000,000^{\circ}$.

Certain minor complications, such as have already been mentioned, do not affect the occurrence of the processes, but they ensure the stability of the star. A process in which the generation of energy increases rapidly as the temperature increases is usually unstable. For any slight increase of temperature causes an excess generation of energy, which causes further heat, still more generation of energy, and so on indefinitely; the process becomes explosive. But when, to take a single instance, a carbon nucleus has swallowed its first proton and become a nitrogen nucleus of mass 13, it must emit a positron before it is ready to swallow a second proton. This is emitted by a sort of radioactive disintegration and, as an increase of temperature does not increase the rate of radioactive disintegration, the explosive sequence 
of events just mentioned does not occur. An increase of temperature will now merely increase the pressure inside the star, so that the star expands and cools. By this thermostatic mechanism, the star continually adjusts itself so that just enough energy is generated to replace that lost by radiation. From its nature the adjustment cannot usually be instantaneous, so it is perhaps not surprising that the stars which are cooler than 'main sequence' stars are mostly variable stars in which regular and substantial fluctuations of light occur.

Thus we think of the matter of the primeval universe as having first condensed to form separate detached masses, one of which ultimately formed our galactic system. This process may well have taken tens of thousands of millions of years. These distinct masses in their turn broke up into separate stars, taking perhaps hundreds of millions of years to do so. These stars would at first be cool, of low density and of immense size. After contracting for a few million years, they attain a temperature at which the protonproton reaction begins to occur. But at low temperatures this reaction occurs but rarely-too infrequently to make good all the energy lost by radiation. The stars accordingly continue contracting until they attain temperatures at which the protons react to an appreciable extent with the nuclei of light elements. This reaction may supply radiation for a few million years, after which the light elements are used up; then contraction begins afresh and continues until the temperature of $20,000,000^{\circ}$ is reached. The carbon reaction now takes control and can supply radiation for thousands of millions of years if the star is not too massive and luminous. During this period, the proton-proton reaction still occurs, and may provide for an appreciable part of the star's radiation-perhaps for half in the sun, and for even more in less massive stars. Finally, the supply of protons gives out, and the star contracts once morepossibly for hundreds of thousands of millions of years.

At some point in this sequence of events the sun has given birth to planets; at some point other stars may do the same. Mathematical investigation shows that planets can be born out of a star by tidal actionthrough a second star approaching near to it and drawing out a long filament of gas, which then breaks up gravitationally and condenses into separate planets. If the two stars are of similar build, such an event requires an approach to within about three radii, whatever the sizes of the stars may be.

Approaches of the requisite closeness would clearly be far more frequent in the early period when the stars were big, than in the later period when they were compact. For our sun, the first period was short (less than 20 million years), while the latter period/ has been long (more than 2,000 million years). Nevertheless, calculation shows that an effective tidal encounter is far.more likely to have occurred in the former short period than in the latter long period. It is not surprising, then, that the solar system is found to contain intrinsic evidence that its planets were born while the sun was still of immense size. It must have extended an appreciable fraction of the distance to the present orhit of Pluto : otherwise there would have been no mechanism for putting the outermost planets in their present orkits.

It follows from this that the age of the sun as a star is approximately equal to the age of the earth as a planet-probably between two and three thousands of millions of years. In this period, the sun can only have used up a small fraction of its protons, so that it probably can look forward to a life in its present state which will be incomparably longer than the life that lies behind it. When this is over, it will begin to shrink into a smaller and cooler star, and life on earth will become impossible.

Detailed calculations show that the chance that a star shall give birth to planets while it is of the compact dimensions of our sun is quite negligible. On the other hand, the chance of its having given birth to planets before attaining to this state is considerable-a fair proportion of the stars must, then, be accompanied by planets. Of these a substantial fraction are likely to be in a physical state not very different from that of our own earth, and so capable of maintaining life like our terrestrial life ; it is possible that such life is far more abundant in space than we used to think.

\section{PREVENTION AND TREATMENT OF ANTI-SOCIAL BEHAVIOUR} $\mathrm{I}_{\text {Prevention and Tecture on "The Differentiation, }}^{\mathrm{N} \text { his Chadwick Leatment of Anti-Social Be- }}$ haviour Disorders" delivered on December 8, Dr. Norwood East has made a succinct but systematic review of the main established facts regarding the causation of crime, and has presented a convincing argument for the view that crime is as much a scientific as a legal or a medical problem. Dr. East was for many years one of H.M. Commissioners of Prisons, and Medical Inspector of Prisons in England and Wales. He has recently completed a scientific study on an extensive scale of the medical, social and psychological aspects of crime, more particularly among adolescents (see Nature, September 26, p. 361). $\mathrm{He}$ is therefore able to speak both with experience and authority; and much of his lecture is in fact concerned with the practical corollaries to be deduced from the inquiries that he has made.

Generally speaking, Dr. East regards behaviour disorders as manifesting in nearly every case a complex causation. They are "the result of stress, acting upon an inherited constitutional pattern, which may itself have been modified, in varying degrees, by. environmental factors other than the stress itself". The inherited constitutional factors are themselves incapable of being greatly changed. It follows that, in general, the treatment of anti-social behaviour must be limited to efforts at changing the environmental factors, and particularly to relieving whatever stress may have precipitated the criminal action. In different cases, however, the relative importance of the constitutional factors, on one hand, and of the environmental factors, on the other, may differ widely. Thus, although individuals belonging to certain temperamental types may be more liable to yield to temptation than persons of average mental heredity, nevertheless, "the majority of persons, and perhaps all, are potential first offenders"; for criminal action, as a rule, results, not from one type of factor to the exclusion of the other, but from "a disturbance of the balance between the two".

According to Dr. East, therefore, the fundamental psychological premise from which the criminologist has to start is the fact that "no person corresponds exactly to his fellows; for even an arbitrarily selected normal person occasionally shows peculiarities of behaviour". Consequently, while anti-social behaviour 